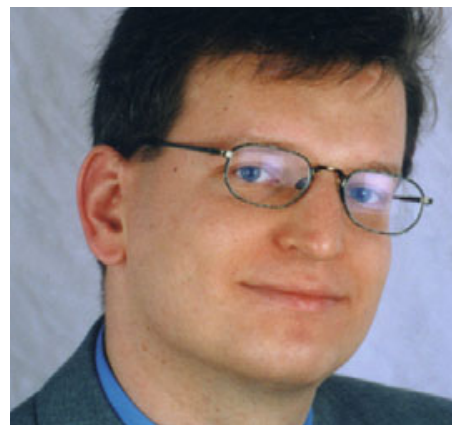

Prof. Dr. med. habil. Jörg Klewer, Zwickau

Schriftleitung

heilberufescience@springer.com

HeilberufeSCIENCE 2011; 2 (1): 2 DOI 10.1007/s16024-011-0103-y

\title{
Erste Listungen
}

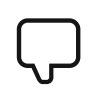

Eine erfreuliche Nachricht: HeilberufeSCIENCE hat seit Ende 2010 erste Zusagen zur Aufnahme in Literaturdatenbanken, wie zum Beispiel CareLit ${ }^{\circledast}$. Weitere sollen in Kürze folgen. Möglich wurde dies, nachdem HeilberufeSCIENCE mit der Ausgabe 1/2010 erstmals als eigenständiges Online-Journal mit eigener Internationaler Standardseriennummer (ISSN) live ging.Weg vom Supplement hin zum eigenständigen Journal: Dieser Schritt war eine der Voraussetzungen für die Aufnahme in eine Literaturdatenbank. Da wir dieses Ziel von Beginn an im Auge hatten, wurde der Aufbau des Journals und der Artikel entsprechend konzipiert: So müssen beispielsweise ein deutsches und englischsprachiges Abstract Bestandteil aller zur Publikation eingereichten Arbeiten sein. Auch die zusätzliche Erklärung zum Ausschluss eines Interessenkonfliktes (Conflict of Interest) durch die einzelnen Autoren ist erforderlich. Natürlich streben wir mittelfristig auch die Aufnahme von Heilberufe-SCIENCE in die Literaturdatenbank MEDLINE ${ }^{\oplus}$ an.

In der Februar-Ausgabe 2011 werden Ihnen nun fünf Artikel und ein wissenschaftlicher Kurzbeitrag geboten. Wir starten mit einem Beitrag von Golombek \& Fleßa, der sich mit Einflussfaktoren auf die Verweildauer im Beruf und die Standortwahl des Arbeitsplatzes bei Gesundheitsund Krankenpflegern befasst. Vor dem Hintergrund des sich abzeichnenden Fachkräftemangels in der Pflege untersucht der Beitrag auch den Einfluss von nicht-monetären Faktoren auf den vorzeitigen Berufsausstieg. Der Artikel von K. Schneider et al. hat eine Untersuchung aus gesundheitswissenschaftlicher Sicht zu Standorten,
Einsatz und Wahrnehmung von automatisierten externen Defibrillatoren zum Thema. Am Beispiel der Großstadt Dresden liefert er jedoch auch für andere Städte wichtige Erkenntnisse zur logistischen Optimierung der Ersten Hilfe bei kardiologischen Notfällen.

Daran an schließt sich die Übersichtsarbeit von V. Schneider et al. „Sterben von Menschen mit Demenz aus Sicht pflegender Angehöriger" und ein pflegepädagogischer Beitrag von Meixner \& Schüßler, der den Wandel der Kommunikation in der Pflegeausbildung analysiert. Den Abschluss bildet der eher methodisch orientierte Beitrag von Hofheinz et al. zur Einschätzung von Sturzgefahren, am Beispiel des Einsatzes des "Timed Up and Go-Test". Das Thema Sturz und Sturzprophylaxe aus praktischer Sicht wird in der wissenschaftlichen Kurzmitteilung von Adner nochmals aufgegriffen. Sie setzt sich mit der Auswertung von Sturzereignissen in einer stationären Pflegeeinrichtung und der Aussagekraft des Tinetti-Tests auseinander.

Jetzt möchte ich Sie wieder zu einer kritischen Lesung einladen, um mit uns in den wissenschaftlichen Dialog einzutreten. Und natürlich würde es mich freuen, Sie in der nächsten Ausgabe von HeilberufeSCIENCE wieder an dieser Stelle begrüßen zu können.

Ihr
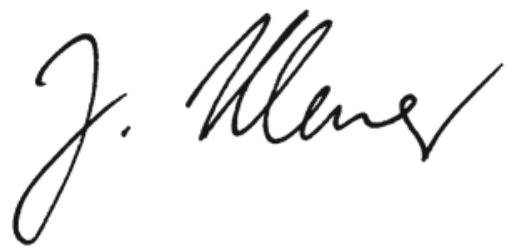